\title{
Different magnetospheric modes: solar wind driving and coupling efficiency
}

\author{
N. Partamies ${ }^{1}$, T. I. Pulkkinen ${ }^{1}$, R. L. McPherron ${ }^{2}$, K. McWilliams ${ }^{3}$, C. R. Bryant ${ }^{3}$, E. Tanskanen ${ }^{4}$, H. J. Singer ${ }^{5}$, \\ G. D. Reeves ${ }^{6}$, and M. F. Thomsen ${ }^{6}$ \\ ${ }^{1}$ Finnish Meteorological Institute, Helsinki, Finland \\ ${ }^{2}$ University of California, Los Angeles, USA \\ ${ }^{3}$ University of Saskatchewan, Saskatoon, Canada \\ ${ }^{4}$ University of Bergen, Bergen, Norway \\ ${ }^{5}$ NOAA, Space Weather Prediction Center, Boulder, CO, USA \\ ${ }^{6}$ Los Alamos National Laboratory, NM, USA
}

Received: 21 March 2009 - Revised: 28 August 2009 - Accepted: 10 November 2009 - Published: 16 November 2009

\begin{abstract}
This study describes a systematic statistical comparison of isolated non-storm substorms, steady magnetospheric convection (SMC) intervals and sawtooth events. The number of events is approximately the same in each group and the data are taken from about the same years to avoid biasing by different solar cycle phase. The very same superposed epoch analysis is performed for each event group to show the characteristics of ground-based indices (AL, PCN, PC potential), particle injection at the geostationary orbit and the solar wind and IMF parameters. We show that the monthly occurrence of sawtooth events and isolated non-stormtime substorms closely follows maxima of the geomagnetic activity at (or close to) the equinoxes. The most strongly solar wind driven event type, sawtooth events, is the least efficient in coupling the solar wind energy to the auroral ionosphere, while SMC periods are associated with the highest coupling ratio $\left(\mathrm{AL} / E_{Y}\right)$. Furthermore, solar wind speed seems to play a key role in determining the type of activity in the magnetosphere. Slow solar wind is capable of maintaining steady convection. During fast solar wind streams the magnetosphere responds with loading-unloading cycles, represented by substorms during moderately active conditions and sawtooth events (or other storm-time activations) during geomagnetically active conditions.
\end{abstract}

Keywords. Magnetospheric physics (Magnetosphereionosphere interactions; Solar wind-magnetosphere interactions; Storms and substorms)

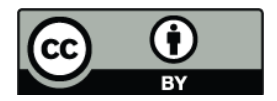

Correspondence to: N. Partamies (noora.partamies@fmi.fi)

\section{Introduction}

Substorms are probably the most common type of magnetic activity. They are an important part of energy circulation through the magnetosphere including reconnection at the dayside magnetopause, storage of energy in the magnetotail and release of the tail energy while reconfiguring the stretched magnetotail into a more dipolar shape. The typical length of the substorm cycle is about $2-4 \mathrm{~h}$ (Tanskanen et al., 2002). Substorms are referred to as isolated non-stormtime substorms when they occur outside storm periods and follow after relatively quiet magnetic conditions. In this case, it is often possible to track the triggering mechanism and energy flow in more detail. More complex substorm events take place during magnetic storms $\left(D_{s t} \leq-50 \mathrm{nT}\right)$ (Kallio et al., 2000).

Steady magnetospheric convection (SMC (Sergeev et al., 1996)) events (also called convection bays (Pytte et al., 1978), continuous magnetospheric dissipation (CMD (Tanskanen et al., 2005)) events, or Balanced Reconnection Intervals (BRI (DeJong et al., 2009))) are periods during which the driving solar wind is steady and the ionospheric convection is enhanced but substorm activity is not observed (McPherron et al., 2005; Sergeev et al., 1996). The solar wind speed is typically rather low, and the magnitude of the interplanetary magnetic field (IMF) is moderate and stable. To distinguish these events from the other low-activity conditions, the minimum duration of an SMC event is usually required to be $3-4 \mathrm{~h}$ (Sergeev et al., 1996), which is longer than the typical time $(2-3 \mathrm{~h})$ between recurring substorms (Borovsky et al., 1993), but comparable to the duration of an average substorm. McPherron et al. (2005) suggest that SMC

Published by Copernicus Publications on behalf of the European Geosciences Union. 
events are periods when reconnection near the sub-solar region at the dayside magnetopause is balanced by tail reconnection in the nightside - a scenario that was speculated on by Pytte et al. in 1978. Later on, Tanskanen et al. (2005) brought into the discussion the pressure balance in the magnetotail. They did not find differences between continuous dissipation and loading-unloading events in the steadiness of the plasma sheet flows but rather in the standard deviation of the total tail pressure, which was lower in case of the SMC-type continuous dissipation than during the loadingunloading cycles.

SMC events often begin with a substorm (e.g. McPherron et al., 2005) - that feature is thought to be related to the pre-conditioning of the magnetosphere for the steady convection (Sergeev et al., 1996). O'Brien et al. (2002) suggested that the solar wind and IMF also have an important effect in the pre-conditioning process, because prior to an SMC event the magnetosphere is usually moderately driven while the magnetosphere prior to an average (isolated) substorm is often quiet. Most SMC periods also end with a substorm (e.g. McPherron et al., 2005). Many recent studies agree that SMCs form a specific group of events with a distinct response of the magnetosphere to the solar wind driving (e.g. Sergeev et al., 1996).

Sawtooth events have also been reported as a separate class of magnetospheric activations. These activations are largeamplitude oscillations of energetic particle fluxes at geosynchronous orbit, recurring with a period of about 2-4 h (e.g. Henderson et al., 2006). The events typically occur during geomagnetic storms when the solar wind driving is strong and the IMF is continuously southward for an extended period of time. A characteristic of these events is that the geosynchronous magnetic field can become highly stretched not only in the midnight sector but also in the evening sector reaching all the way to the dusk meridian (Pulkkinen et al., 2006; Reeves, 1994). This can be observed as a reduction of the magnetic field inclination at geosynchronous orbit over a wide range of local time sectors, as well as in the strongly enhanced partial ring current as measured by the ASY-H index. It has also been suggested that sawtooth events are, in fact, recurring quasi-periodic substorms during storm-times (Henderson et al., 2006). And as pointed out by Reeves et al. (2004), sawtooth events are interesting because they are an intermediate state between isolated non-storm substorms and full-blown storm-time activity.

A recent study by Pulkkinen et al. (2007) presented a statistical comparison of the typical solar wind driver conditions and ionospheric activity for sawtooth events and substormlike auroral electrojet activations during geomagnetic storms. They concluded that sawtooth events are not a specific type of magnetic activity, and that the $2-3 \mathrm{~h}$ periodicity, strong stretching of the dusk sector field, and strongly asymmetric ring current are also found in association with other types of storm-time activations. Furthermore, they demonstrated that the level of driving is very similar during the sawtooth events and other storm-time activations, while the auroral activity (AL index) is slightly lower in the case of sawtooth events.

In this paper, we perform analysis similar to that of Pulkkinen et al. (2007) to SMC events and isolated substorms. Our aim is to explore the differences among sawtooth events, SMC intervals and isolated non-stormtime substorms in both the driving conditions as well as ionospheric and magnetospheric activity. Here, we use the sawtooth events as representatives of storm-time activations, because they are generally easier to list, identify and agree on than any other type of storm-time events.

\section{Event selection and data}

\subsection{Event classification}

We used a data set of 138 sawtooth events (1999-2002) compiled by R. L. McPherron as a representative of storm-time activations. Sawtooth events were visually identified as recurring, relatively dispersionless particle injections observed by multiple geosynchronous satellites in multiple magnetic local time sectors (Pulkkinen et al., 2007). In this study, we consider individual sawteeth as separate events.

SMC events were identified from the AE index data from the time period of 1998-2001. These years were selected to be close to the period from which the sawtooth event data were compiled, and thus, to reduce any bias due to solar cycle variations in the ionospheric and solar wind driving conditions. An automated selection procedure required that during the SMC period the auroral electrojet activity is at the level of $\mathrm{AE}>200 \mathrm{nT}$ and that the AL index is changing at a rate slower than $25 \mathrm{nT}$ per $\min (\mathrm{dAL} / \mathrm{dt}>-25 \mathrm{nT} / \mathrm{min})$ (O'Brien et al., 2002; McPherron et al., 2005). The threshold for the $\mathrm{AE}$ index has been chosen so that the auroral activity level is well above the quiet time values. The AL gradient restriction is applied to eliminate substorm occurrence (abrupt decreases of AL) during the steady convection events. In addition, the above criteria were required to hold for at least three hours. As a result, we found 149 SMC events during the four-year period.

Since most SMCs begin with a substorm, the substorm onset was chosen to be the reference time for our analysis. The AL index curve for each automatically found convection period was visually inspected in order to find the onset of the substorm that initiates the SMC. The onset was defined as an abrupt decrease of at least $100 \mathrm{nT}$ in the $\mathrm{AL}$ index within three hours from the beginning of the SMC interval. The SMC event was taken to follow directly from the substorm recovery. In $56 \%$ of the cases the automatically detected SMC period starts within one hour after the onset. 27 events were discarded because a substorm could not be identified prior to the SMC.

Substorms analysed in this study have been selected from the vast set of events that were visually identified as 
brightenings of the aurora in the IMAGE satellite data (Frey et al., 2004). For our purposes, we chose a subset of 155 substorms that were also observed in the AL index data. The selection criterion was an abrupt decrease of at least $100 \mathrm{nT}$ at the time of the onset that led to a negative bay development. These substorms are from the time period of 2000-2002.

\subsection{Data sets}

The same data sources and analysis methods were used for all three event groups. For the 138 sawtooth events, 122 SMC events and 155 substorms, the IMF and solar wind parameters were examined to determine the driving conditions. The ionospheric activity is characterized by the auroral electrojet index (AL), symmetric and asymmetric ring current indices (SYM-H and ASY-H), northern polar cap index (PCN) and the cross-polar cap (PC) potential. The magnetospheric behaviour at the geostationary orbit is characterised by the energetic electron fluxes.

The solar wind parameters from Solar Wind Electron Proton Alpha Monitor (SWEPAM) instrument (McComas et al., 1998) and the IMF from the MAGnetic field experiment (MAG) (Smith et al., 1998) instrument, both on board the Advanced Composition Explorer (ACE) satellite were examined. ACE is located at the L1 point roughly $220 R_{E}$ upstream of the Earth. All ACE data have been propagated to the magnetopause (to the distance of $10 \mathrm{R}_{E}$ upstream of the Earth) using the upstream distance of the satellite from the magnetopause and the average solar wind speed during the interval of interest. We use the IMF $X, Y$ and $Z$ components and its magnitude as well as the solar wind number density, dynamic pressure and speed to calculate the epsilon parameter (Akasofu, 1981), as well as the dawn-to-dusk electric field $\left(E_{Y}=-V_{X} B_{Z}\right)$.

The $\epsilon$ parameter (Perreault and Akasofu, 1978) is defined as

$\epsilon=\frac{4 \pi}{\mu_{0}} V_{S W} B^{2} l_{0} \sin ^{4}(\Theta / 2)$,

where $\mu_{0}$ is the vacuum permeability, $V_{S W}$ is the solar wind speed, $B$ is the magnitude of the IMF, $l_{0}=7 R_{E}$ is an empirical scaling parameter, and $\tan (\Theta)=B_{Y} / B_{Z}$ determines the IMF clock angle. All variables in the equation are given in GSM coordinates and SI units.

The global AL index is used whenever available. For those events for which the global AL was not yet available (46 sawtooth events in 2002), a quasi-AL index was calculated from the magnetic recordings of the ground-based networks IMAGE (Viljanen et al., 1997) in Fennoscandia and CARISMA (old CANOPUS, Rostoker et al., 1995) in central and western Canada. Thus, the quasi-AL only records substorm activity in and around these two local time sectors. The symmetric and asymmetric parts of the ring current are described by SYM-H and ASY-H indices, respectively. They are calculated as weighted averages (SYM-H) and maximum differences (ASY-H) of 4-6 mid-latitude stations around the globe
(Iyemori, 1990; Sugiura and Kamei, 1991). The PCN index is constructed from magnetic recordings at the Thule station located within the polar cap in Greenland (Troshichev et al., 1979, 2000). This index is generally used as a proxy for the ionospheric convection and thus, the reconnection rate. PC potential values were estimated from the Super Dual Auroral Radar Network (SuperDARN, Greenwald et al., 1995) measurements. Spherical harmonics are fitted to the recorded convection velocities to produce a smooth convection map over each hemisphere. The difference between the maximum and minimum voltages in the convection pattern is used as an estimate of the cross-polar cap potential (Ruohoniemi and Baker, 1998).

The temporal resolution of the ground-based indices is one minute, except the PC potential which is calculated once every two minutes. For all ACE parameters we use 64-s data.

The geostationary orbit electron fluxes were obtained from the Los Alamos National Laboratory satellites. Synchronous Orbit Particle Analyzer (SOPA) data from the geostationary satellites 1989-046, 1990-095, 1991-080, 1994-084, LANL97A, LANL-01A, and LANL-02A (Belian et al., 1992) are used to monitor the magnetospheric activity. Electron flux enhancements and injections were examined in the energy range from 50 to $315 \mathrm{keV}$ (the five lowest channels), corresponding to typical substorm injection energies.

\section{Statistical comparison}

Sawtooth events occur during magnetic storms. During the sawtooth events, the $D_{s t}$ index typically varied between $-140 \mathrm{nT}$ and $-80 \mathrm{nT}$, which is mainly in the range of intense storms $\left(D_{s t}<-100 \mathrm{nT}\right)$ as defined by Gonzalez et al. (1994). For the SMC events, the peak $D_{s t}$ was found between $-60 \mathrm{nT}$ to $-20 \mathrm{nT}$ showing less ring current activity than during the sawtooth events, but still about half of the events were recorded during moderate storms $(-100 \mathrm{nT}<$ $D_{s t}<-50 \mathrm{nT}$ ). Finally, during most of the substorms the $D_{s t}$ varied between $-40 \mathrm{nT}$ and $0 \mathrm{nT}$, which is the tail of weak storms $\left(-50 \mathrm{nT}<D_{s t}<-30 \mathrm{nT}\right)$. The $D_{s t}$ peak of about $-30 \mathrm{nT}$ is also given as a typical substorm value by Gonzalez et al. (1994).

As a measure of the global activity and magnetospheric convection (Thomsen, 2004), we present the normalised distribution of the $K_{p}$ index values during sawtooth events (brown/red), steady magnetospheric convection events (orange/yellow) and substorms (green/light green) in Fig. 1. For the sawtooth events and substorms, the $K_{p}$ value is the threehour value closest to the individual sawtooth or substorm onset. For the SMCs, the $K_{p}$ indices are selected from the middle of the steady convection period. The peak $K_{p}$ values for the SMCs and substorms are 1-3, while the ones for the sawtooth events range from 4 to 6 . The tails of these distributions do overlap, but the average activity, and thus convection level, during the sawtooth events is clearly higher 


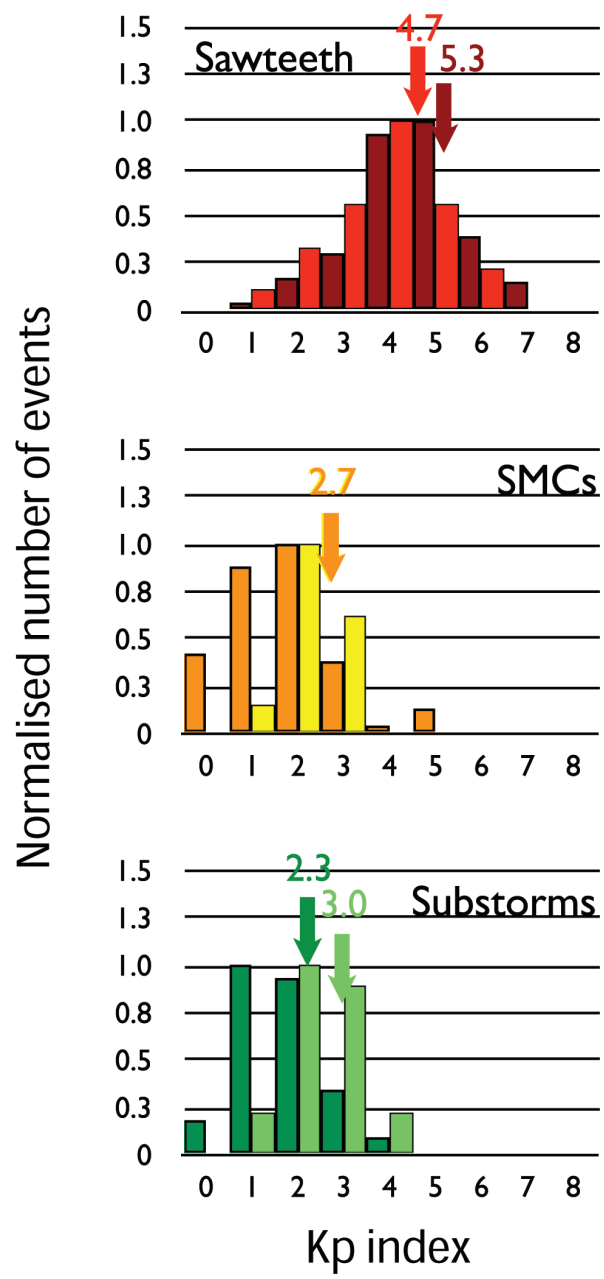

Fig. 1. Distribution of the global activity in terms of $K_{p}$ index. $K_{p}$ value is binned on $\mathrm{X}$ and a normalised (described below) number of events on Y axis. The middle panel distribution represents the $K_{p}$ values recorded in the middle of the SMC periods (orange/yellow), while the top and bottom distributions show the $K_{p}$ values at the time of the sawtooth (brown/red) and substorm events (green/light green), respectively. The lighter coloured histograms represent the subsets of constant solar wind electric field. The arrows and their labelling numbers are the median values of each distribution. All histograms are normalised to overcome the different number of events in subsets of each event group.

than the activity during the SMCs and substorms. The $K_{p}$ distribution for SMCs is, on average, similar to the ones for substorms.

A clear difference between these event groups is the seasonal variation in their occurrence. This is demonstrated by the monthly distribution on the left hand side of Fig. 2. While the SMC events (yellow) are most frequent during the summer months (April to July), the sawtooth events (red) are mainly observed around the autumn and spring equinoxes during which the substorm occurrence minimizes. The oc- currence of the sawtooth events is in agreement with the seasonal behaviour of the geomagnetic activity and occurrence of the aurora (e.g. Nevanlinna, 2004): minima around the solstices in July and December, maxima close to the equinoxes in March and September, although the spring maximum in this data set is less pronounced. This semi-annual variation has often been explained by the Russell-McPherron mechanism, where the activity occurrence maxima correspond to the periods of largest component of the IMF being antiparallel with the dayside geomagnetic field (Russell and McPherron, 1973). The summer maximum of the SMC periods is most likely due to the fixed threshold value of the $\mathrm{AE}$ index in the selection criteria. During summer months the ionospheric conductivity is higher, the auroral electrojets are stronger, and the AE index threshold is more frequently exceeded. These data sets indicate that the sawtooth events are more concentrated around periods of highest geomagnetic activity close to the equinoxes, while the SMC events and substorms are more evenly distributed throughout the year. The tendency for fewer substorms and more sawtooth events during the equinoxes may indicate that the higher level of geomagnetic activity turns substorms into storm-time activations, such as sawteeth. As a reference, the right hand column of Fig. 2 contains the average monthly distribution of $K_{p}, \mathrm{AE}$ and $D_{s t}$ indices. The index data are constructed from daily averages for the time period 1998-2001. $K_{p}$ and $D_{s t}$ variations show the changes in the average geomagnetic activity, which is also reflected by the sawtooth event observations. The AE distribution demonstrates the effect of the higher ionospheric conductivity in summer months.

\section{Responses at geostationary orbit}

The results of the superposed epoch analysis of the geosynchronous particle fluxes for three local time sectors, evening sector (18:00-22:00 MLT), midnight sector (22:00-02:00 MLT) and morning sector (22:00-06:00 MLT) are shown in Fig. 3. About $50 \%$ of the isolated substorms (60-80 events) occurred at the time when there was a LANL satellite located in at least one of these sectors and taking data. Particle injections related to the isolated substorms (left column) are typically transient and their timing does not exactly match the onset timing determined from the groundbased data, which results in a smooth and gentle changes in the epoch fluxes around the onset times. Furthermore, for about one third of the isolated substorm events no injection was observed at the geostationary orbit. The level of the electron fluxes near the zero epoch time is slightly lower for the isolated substorms than it is for the substorms prior to SMCs (middle column). In the morning sector, the fluxes at the lowest energy channels maximize about an hour after the onset. The latest flux increase is seen in the evening sector (the lowest energy channel) about two hours after the onset, which suggests an electron drift from the midnight sector injection. 

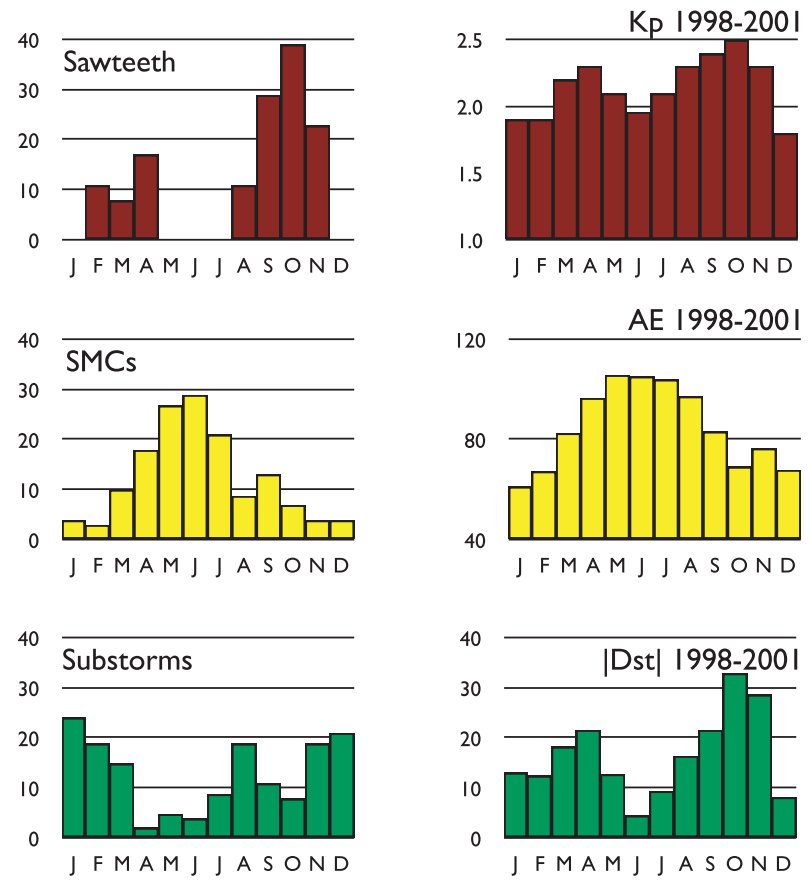

Fig. 2. Monthly distribution of the sawtooth events, SMCs and substorms (left column). The middle panel distribution represents the monthly occurrence of the SMC periods, while the top and bottom panel distributions show the monthly occurrence of the sawtooth events and isolated substorms, respectively. For a comparison, the right hand column contains the average monthly distribution of $K_{p}$, $\mathrm{AE}$ and $D_{s t}$ indices. The index data are constructed from daily averages of the time period 1998-2001.

Although the zero epoch for SMCs is chosen to be the substorm onset prior to the steady convection interval, there is only a slight change in the midnight sector fluxes, while a slightly larger flux increase is observed in the morning sector, again strongest in the lowest-energy channel. This would indicate that much like the isolated substorms, the substorms initiating the SMC periods are not very strong, are not associated with significant electron energization, and rarely intrude into the geostationary orbit. For SMCs, no dipolarization signatures were evident in the superposed epoch results of the magnetic field inclination at the geostationary orbit (data not shown). Typically no substorm type particle injections were observed during the SMC periods (data not shown).

In strong contrast to the SMC and isolated substorm events, the sawtooth events are associated with (and defined by) very strong geostationary orbit particle injections. The electron fluxes for the sawtooth events in three different time sectors are given in the right panels of Fig. 3. It can be seen that the fluxes prior to the sawtooth events are similar to those measured during the other events, but the strong geosynchronous particle injections at sawtooth onsets are seen at all local time sectors nearly simultaneously. This highlights the fact that the sawtooth events are associated with processes that occur over a wide local time sector in the neargeostationary region.

\section{Coupling efficiency}

The coupling efficiency between the solar wind and the auroral ionosphere parametrized by $\mathrm{AL}-$ to- $E_{Y}$ and PC-to- $E_{Y}$ ratios have been calculated for the different event groups. Here, the absolute values of $\mathrm{AL}, \mathrm{PC}$ and $E_{Y}$ are used to calculate the ratios. The ratios have been determined for each event separately, and Fig. 4 contains superposed epoch curves based on single event coupling efficiencies. The superposed epoch analysis was performed for the three different event groups. The zero epoch time was chosen to be the individual onset time for isolated substorms and sawtooth events, while the onset of the substorm prior to the SMC period is the zero epoch time for SMCs.

The sawtooth events (black curves) are much more strongly driven compared to the SMCs and substorms (with average $E_{Y}$ values of $3.4 \mathrm{mV} / \mathrm{m}, 1.1 \mathrm{mV} / \mathrm{m}$ and $0.6 \mathrm{mV} / \mathrm{m}$, respectively). The average $\mathrm{AL}$ index and polar cap potential values reflect the same ordering in the ionosphere. The coupling efficiency as defined by $\mathrm{AL} / E_{Y}$ for the different event types shows a different behaviour: sawtooth events are the least efficient in using the energy provided by the solar wind. The coupling ratio only reaches about 150 around the peak of a sawtooth injection (black line). The coupling efficiency for isolated substorms (green line) peaks higher, around 180200, at the substorm onset time. The most effective coupling of 200-220 is found during the substorms prior to steady convection periods (blue line). During the convection period itself (epoch time 2-4), the coupling efficiency still remains at the sawtooth peak value level. This suggests that the weaker the driver the better the coupling is. The most efficient energy coupling interval in the substorm case is very short-lived as compared to the steady high efficiency of sawtooth events, and especially the high efficiency of SMCs that lasts for hours.

The PC potential related coupling efficiency, although much steadier, suggests a similar conclusion. The weak solar wind electric field couples very effectively into the auroral ionosphere, while much of the energy of the strong driving during sawteeth is lost in the interaction.

\section{Effect of the driving solar wind electric field}

Epoch curves for a set of selected IMF, solar wind parameters and ground-based indices are plotted in Fig. 5. This figure shows the IMF $B_{Z}$, solar wind dynamic pressure, solar wind speed, epsilon parameter, electric field and AL index epoch curves for sawtooth events (black), SMCs (blue) and isolated substorms (green). The IMF $B_{Z}$ decreases in a similar manner for all event types prior to the zero epoch 

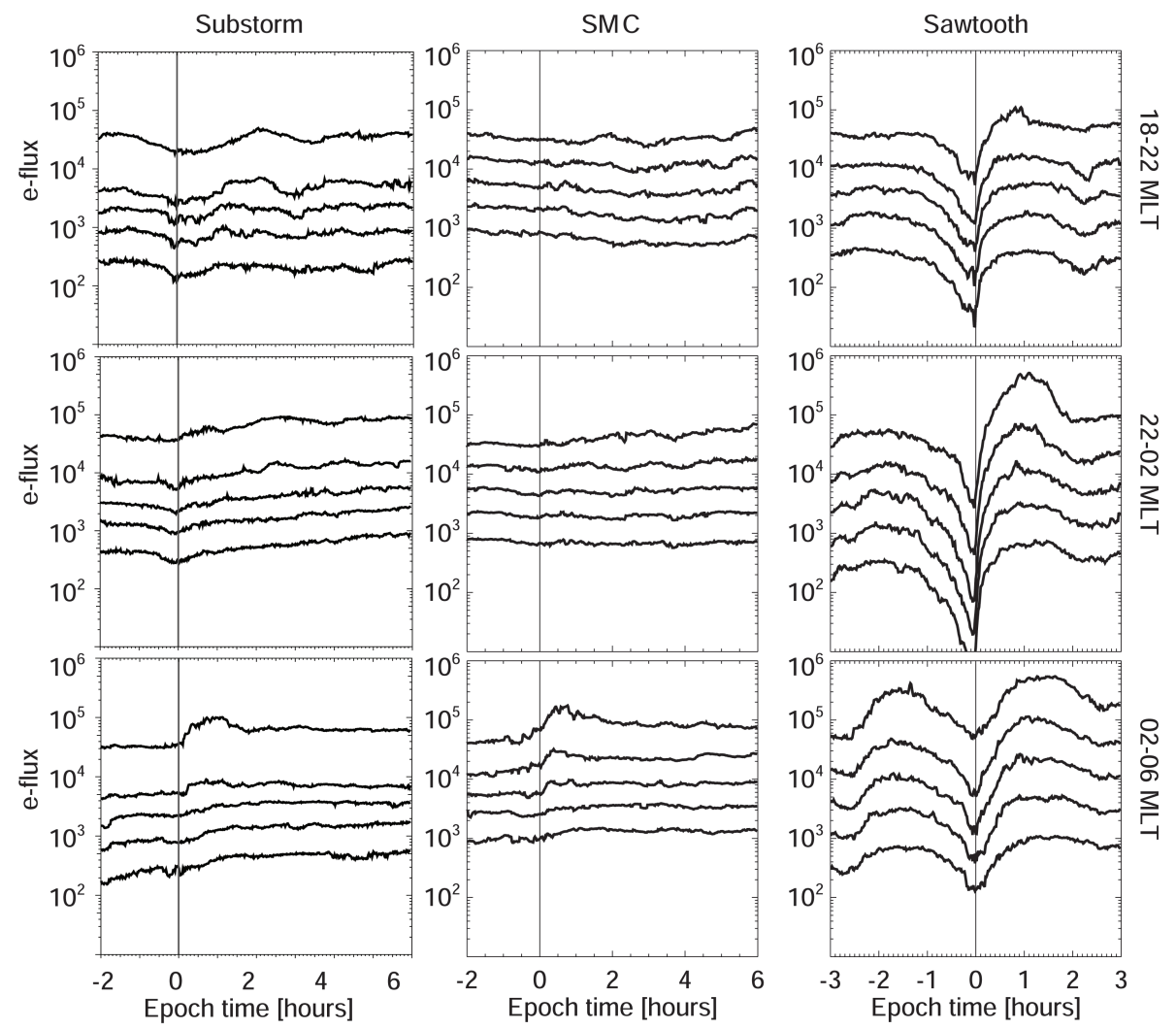

Fig. 3. Superposed epoch analysis results of the geosynchronous electron fluxes at 18:00-22:00 MLT, 22:00-02:00 MLT, and 02:0006:00 MLT for the isolated substorms (left panels), SMC events (middle panels) and for the sawtooth events (right panels). The electron fluxes are shown in the range 50-315 keV (five lowest energy channels) in units of $1 / \mathrm{cm}^{2} / \mathrm{s} / \mathrm{sr} / \mathrm{keV}$. The vertical black line marks the zero epoch, which is defined as the ground-based substorm onset for the SMC periods and the geostationary orbit injection onset for the sawtooth events.

time. The negative $B_{Z}$ remains at the same level for sawtooth and SMC events after the onset, but recovers back to the presubstorm level for the isolated substorms. The pressure and the speed are typically very steady and the differences between the groups are rather small, except the speed is high for sawteeth. The energy inflow into the magnetosphere is at the storm values (1 TW) for sawtooth events but much less for SMCs and substorms. The solar wind electric field and the AL index show the same ordering of the event groups as does the IMF $B_{Z}$. In addition, the AL index epoch experiences some quasi-periodic behaviour during the sawtooth events, while the AL values for SMCs reflect their selection criteria of beginning with a substorm and continuing as an extended recovery phase with an enhanced level of convection.

In addition to the above described event groups, the superposed epoch analysis was also performed for sub-groups of sawtooth, steady convection and substorm events. The sub-groups were selected so that the driving conditions (defined by the solar wind electric field) were most alike. That appears when the solar wind motional electric field was re- quired to be relatively constant and about the same magnitude: $1.5 \mathrm{mV} / \mathrm{m} \leq E_{Y} \leq 2.5 \mathrm{mV} / \mathrm{m}$. The $E_{Y}$ averages were taken over the entire steady convection period for SMCs, and from half an hour before to half an hour after for the individual substorm and sawtooth onsets. This criterion results in a set of 21 substorms, 23 SMCs and 25 sawtooth events, whose epoch plots are shown in Fig. 6. For this subset of sawtooth events, IMF, solar wind, and ground-based parameter values are smaller than what is typical for the entire set of sawtooth events. For this subset of SMCs and substorms, most of the parameters are higher than what is typical for the full set of SMCs and substorms (Fig. 5). This suggests that the solar wind electric field has an important role in setting the type of activity. The median $K_{p}$ index values for the fixed- $E_{Y}$ subsets also show a smaller deviation: 3.0 for the substorms, 2.7 for the SMCs and 4.7 for sawtooth events, compared with the corresponding mean values of 2.3, 2.7 and 5.3, respectively, for the full data sets (see the arrows and the arrow labels in Fig. 1).

The solar wind and IMF data (Fig. 6) reveal interesting differences between these subsets. While the driving electric 


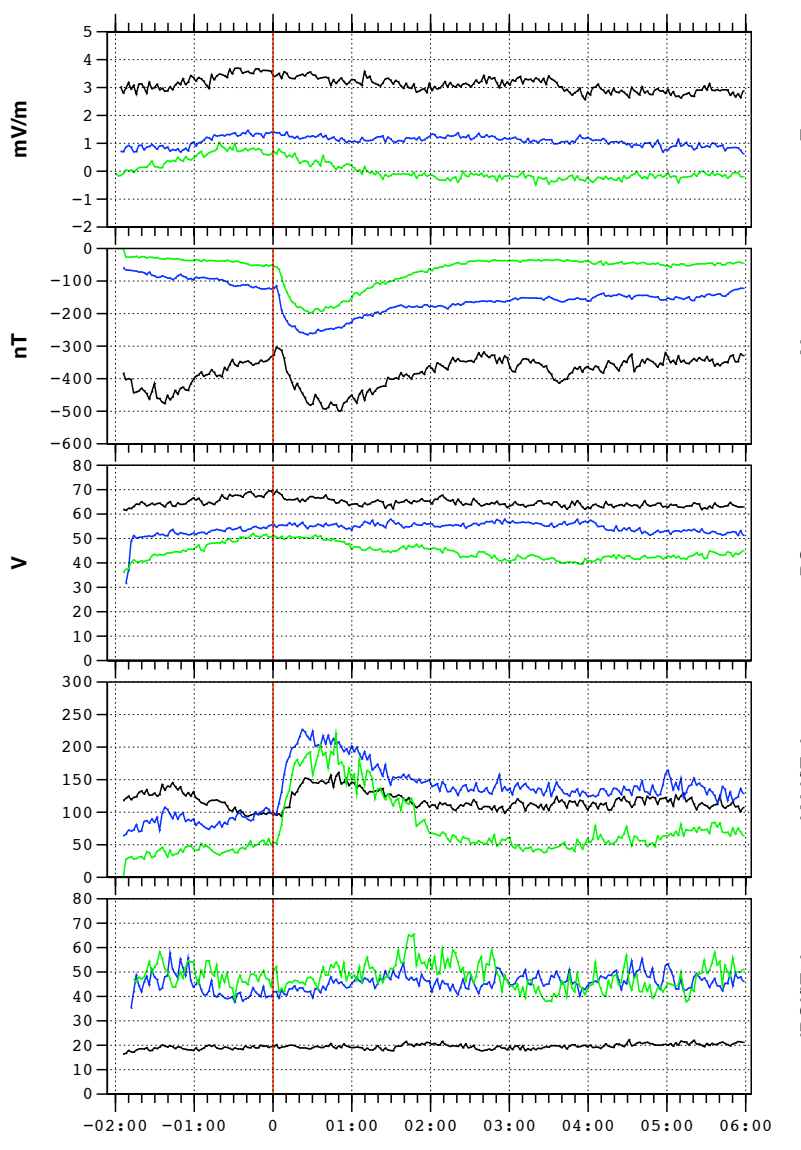

Fig. 4. Superposed epoch curves solar wind $E_{Y}$, AL index, polar cap potential as well as coupling efficiencies as $\mathrm{AL} / E_{Y}$ and $\mathrm{PC} / E_{Y}$ for sawtooth events (black), SMC periods (blue), and isolated substorms (green).

field is almost the same for all subsets (a selection criterion), the constituents of the electric field ( $V_{X}$ and $\left.B_{Z}\right)$ are not. The average solar wind speed is $380 \mathrm{~km} / \mathrm{s}$ for the SMC events, $440 \mathrm{~km} / \mathrm{s}$ for substorms, and $480 \mathrm{~km} / \mathrm{s}$ for the sawtooth events. This is a lower speed for SMCs but a higher speed for substorms than found in the full data set (Fig. 5). The large solar wind speed differences suggest that the magnetosphere is more stable when the solar wind is slow, and the higher speed drives a more dynamic activity.

To take a more detailed look into the velocity differences for the subset of events in Fig. 6 we plot the median and quartile (25\% and $75 \%$ ) solar wind speeds for all three event groups in Fig. 7. In this figure, we have calculated one mean value for each event group and also included a subgroup of storm-time activations. The $E_{Y}$ selection criterion for the storm-time activations was the same as we used for the other groups. A more detailed analysis of the storm-time activations is described by Pulkkinen et al. (2007). The subgroup of stom-time activations consist of 35 events, with a median speed of $570 \mathrm{~km} / \mathrm{s}$. Compared to the full event sets, the selec-

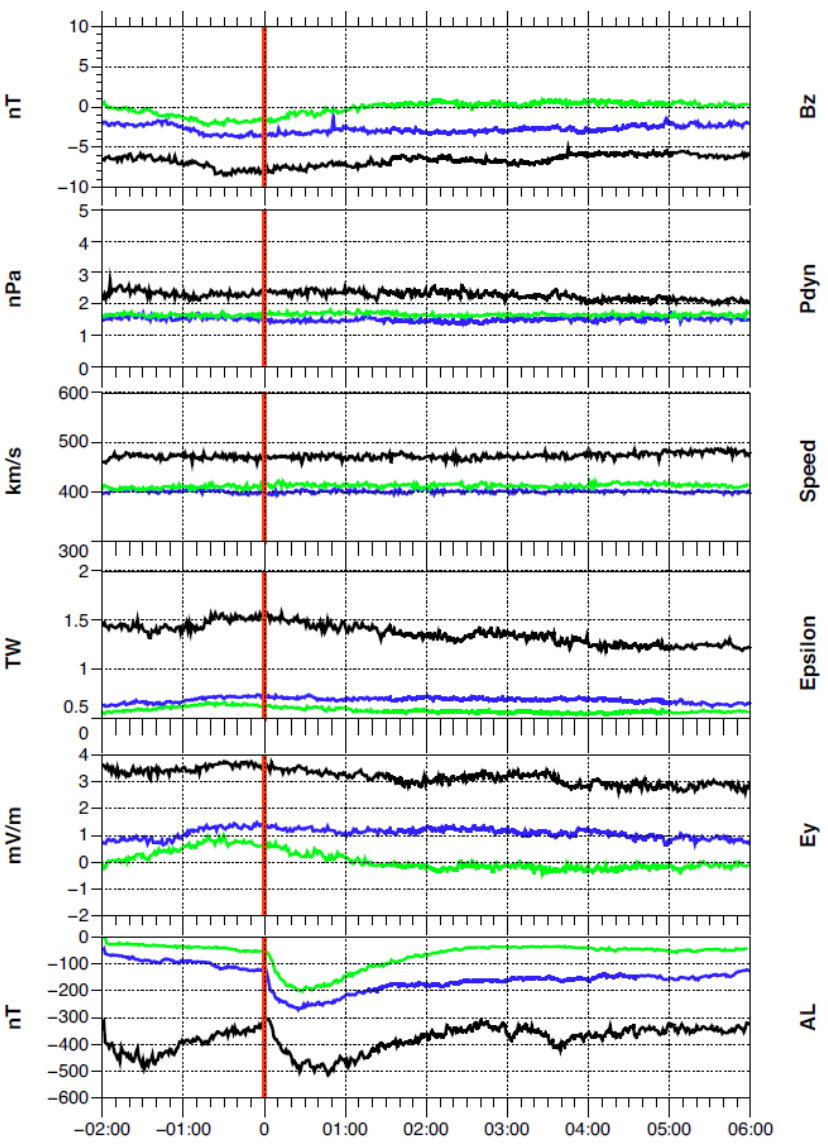

Fig. 5. Superposed epoch curves for the full sets of sawtooth events (black), SMC periods (blue), and isolated substorms (green). The panels from top to bottom are: IMF $B_{Z}$, solar wind dynamic pressure, solar wind speed, epsilon parameter, solar wind motional electric field, and AL index.

tion of subgroups makes the velocity differences smaller between the family of loading-unloading cycles, i.e. substorms, sawteeth and storm-time activations, but larger between the steady convections and the substorms. From the plot it is clear that the higher the speed the larger differences there are in the velocity between single events (separation between the quartiles, i.e. the error bars). The SMC quartiles are much closer to the median velocity than the quartiles of other events. Similar steadiness was recently reported by (DeJong et al., 2009). Furthermore, the quartiles span a much larger range of velocities for sawtooth and storm-time events than they do for the isolated substorms and SMCs. Although the solar wind speed seems to be ordering the magnetospheric response into different event groups, especially the loadingunloading type events (substorms, sawtooth events, stormtime activations) really form a continuum rather than welldefined, separate distributions. It is also interesting to point out that the mean standard deviation for any single event, in any of the event groups, is only $10-20 \mathrm{~km} / \mathrm{s}$. Thus, the solar wind speed is very steady in all of these event groups. 


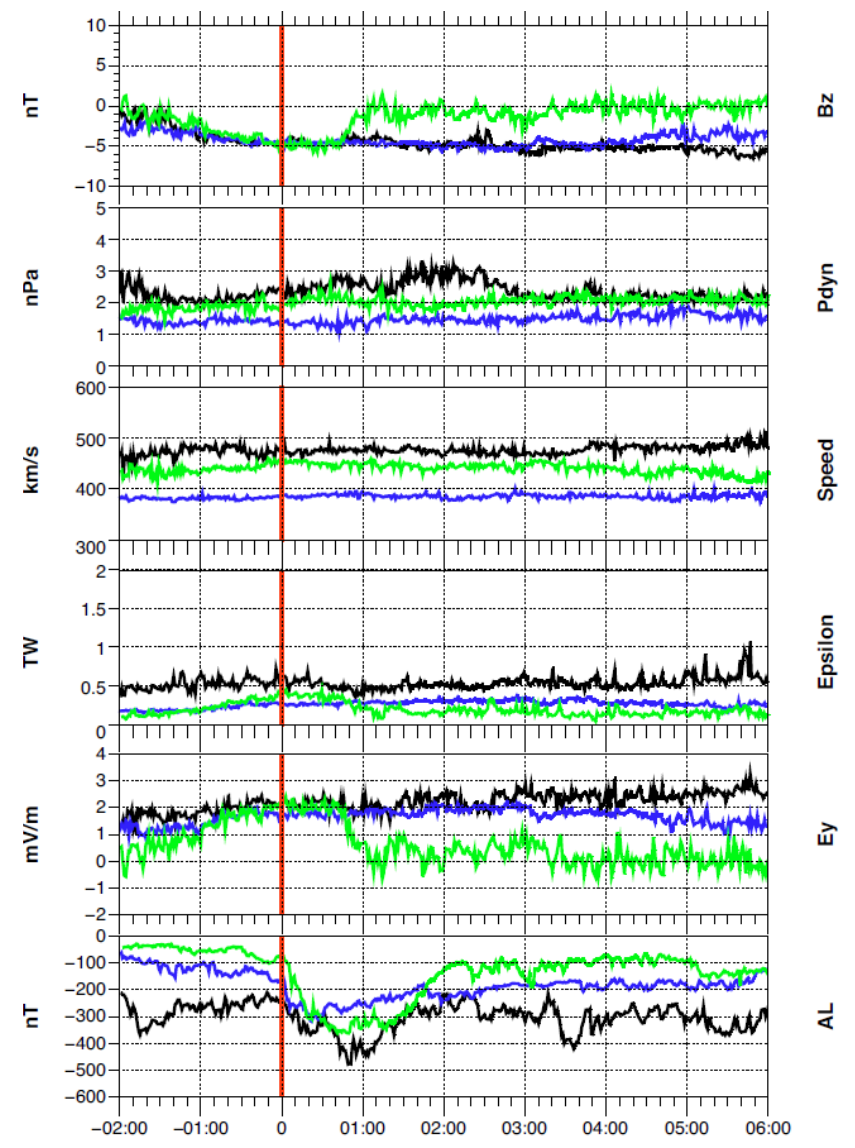

Fig. 6. Superposed epoch curves for the subsets of constant $E_{Y}$ of sawtooth events (black), SMC periods (blue), and isolated substorms (green). The panels from top to bottom are: IMF $B_{Z}$, solar wind dynamic pressure, solar wind speed, epsilon parameter, solar wind motional electric field, and AL index.

\section{Conclusions}

The level of geomagnetic activity as measured by $K_{p}$ index is rather similar for isolated substorms and SMC events (13 ), but clearly higher for sawtooth events (4-6). This reflects the fact that the sawtooth events take place during the magnetic storms, and thus, during stronger magnetospheric convection, while most of the substorms and SMCs are observed during more quiet periods. The monthly occurrence of the isolated substorms and sawtooth events is concentrated near the equinoxes, but the SMC distribution turns out to be much more even. As the SMC distribution has its smooth maximum during the summer months, the maxima for the sawteeth and the minima substorms appear close to the equinoxes where the geomagnetic activity is strong as well. This may suggest that as the geomagnetic activity increases the type of activation observed in the magnetosphere changes from isolated substorms into the storm-time activations.

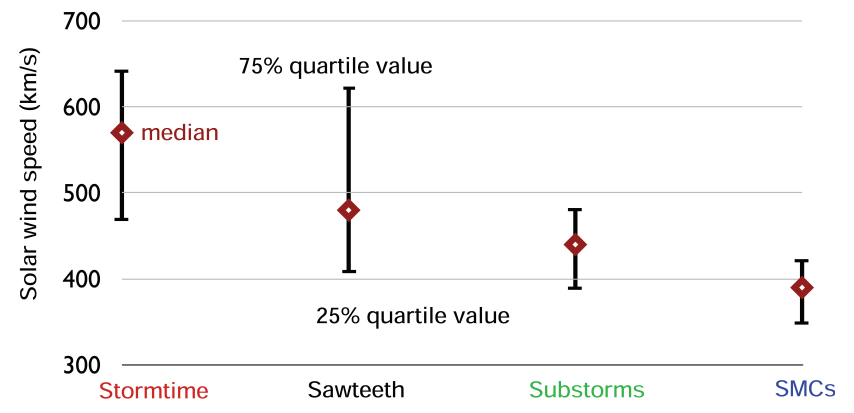

Fig. 7. Median (red diamonds), $25 \%$ and $75 \%$ quartile (black error bars) velocities for the subgroups of SMCs (blue), isolated substorms (green), sawtooth events (black) and storm-time activations (red), for which the driving solar wind $E_{Y}$ ranges from 1.5 to $2.5 \mathrm{mV} / \mathrm{m}$.

The solar wind driving (dawn-dusk electric field and IMF $\left.B_{Z}\right)$ is the strongest for sawtooth events, the second strongest for the steady convections and the weakest over the periods of isolated substorms. However, the solar wind is, on average, very steady for all three event groups. So, as is also suggested by single event data, majority of these events have not been triggered by sudden solar wind variations. Subgrouping the event types according to the average solar wind electric field results in events that appear more similar to each other than the full set of sawteeth, SMCs and substorms. The differences between the three sub-groups in almost any given parameter becomes smaller. But the largest contrast that remains, and even enhances, is the solar wind speed: The fast wind relates to the loading-unloading type events, i.e. sawtooth and isolated substorm events, while the slow solar wind drives steady convection periods in the magnetosphere.

Superposed epoch analysis of the coupling efficiency between the solar wind and the ionosphere $\left(\mathrm{AL} / E_{Y}\right)$ the sawtooth events that are related to the strongest solar wind driving have the weakest coupling to the auroral ionosphere. It turns out that the SMC is the event type that most efficiently uses the solar wind energy. The coupling efficiency during isolated substorm events is very high as well, but the effective coupling interval only lasts for a short while (the expansion phase and early recovery, about $2 \mathrm{~h}$ ) compared to the extended strong coupling (up to $6 \mathrm{~h}$ ) during the SMC events. In fact, it has been shown in an earlier study by Manninen et al. (2008) that SMCs can very efficiently dissipate energy accumulated in the magnetotail during a superstorm (7-8 November 2004). Similar results have been reported from a very different analysis by McPherron et al. (2009), who showed that the weakest coupling appears where the driving is the strongest. Geoefficiency of storms driven by corotating interaction regions (CIRs) and coronal mass ejections (CMEs) were compared by Turner et al. (2009). CIR-driven storms were related to lower IMF $B_{Z}$, smaller values of the epsilon parameter and a less negative $D_{s t}$ peak 
than the CME-driven storms. However, the coupling efficiency of the CIR storms appeared to be higher than the CME storms. Using both their and our event lists, we calculated that during the same years $10 \%$ ( 7 out of 70 ) of CME-driven storms contained steady convection periods, and 17\% (11 out of 66) of CME-driven storms included sawtooth events. Furthermore, $13 \%$ (4 out of 30) of CIR-driven storms contained steady convection periods, and only 6\% (2 out of 34 ) of CIRdriven storms included sawtooth oscillations. This finding further agrees with more geoefficient CIR-driven storms creating more favourable conditions for SMCs, whose coupling efficiency is higher than the coupling during the sawtooth events.

A clear difference between isolated substorms, substorms initiating steady convection intervals, and the onsets of the sawtooth events is their location and size in the magnetotail. While the sawtooth oscillations occur nearly instantaneously in the magnetotail over a wide range of local times, as observed by geostationary satellites (Henderson et al., 2006), the superposed epoch curves for the SMC intervals and isolated substorms indicate that the injections do not always reach geostationary orbit, they are more transient and local, and that they typically appear on the lowest energy channels. Farther down the tail, the plasma sheet can be active during the steady convection periods, as reported by Tanskanen et al. (2005). They studied high-resolution Geotail observations that revealed frequent flow bursts both Earthward and tailward even when the total tail pressure and the ionospheric auroral activity were steady. They also noted that the standard deviation of the tail total pressure is much lower for continuous dissipation (SMC-type) events than for the loadingunloading events. This finding is in agreement with the level of fluctuations in the solar wind (the standard deviation of the solar wind parameters) being much lower for the SMCs than for any other event type (Partamies et al., 2009). Without obvious particle injections at the onset, the isolated substorms may seem like what was described as small substorms during a contracted auroral oval by Petrukovich et al. (2000) and Lui et al. (1976). The average AL peak of $-200 \mathrm{nT}$ for our substorm events is in the range of small substorms by Petrukovich et al. (2000). However, the other criteria for small substorms are not fulfilled by our event group: instead of the PC index staying below unity, we found peak values of 1.5, and instead of the substorms occurring at and north of Bear Island station $\left(71.45^{\circ} \mathrm{MLAT}\right)$ the mean latitude of the onset latitude for our data set is $66.5^{\circ}$ MLAT. As in any set of events, there are vast differences within each event group, but we are very confident that most of the isolated non-storm substorms analysed in this study occur within average oval latitudes and experience a full expansion and recovery phase.

In summary, our statistics show that for the conditions: IMF southward, slow solar wind and less negative $B_{Z}$, the result is a more effective coupling between the solar wind and the auroral ionosphere. The coupling efficiency $\left(\mathrm{AL} / E_{Y}\right)$ during magnetic storms is typically much lower. In addition to the geoefficiency, the solar wind speed plays an important role in ordering the type of magnetospheric response, since steady convection periods are related to low speed values and sawtooth events and storm-time activations to much faster solar wind driving.

Acknowledgements. The work by N.P. was supported by the Academy of Finland. The authors thank Robin Barnes for providing the SuperDARN polar cap potential data. We thank ACE PI teams for the MAG and SWEPAM data. ASY-H/SYM-H and AE indices where downloaded from Kyoto Geomagnetic Data Services, and the PCN data from the Danish Meteorological Institute web site.

Topical Editor I. A. Daglis thanks two anonymous referees for their help in evaluating this paper.

\section{References}

Akasofu, S.-I.: Energy coupling between the solar wind and the magnetosphere, Space Sci. Rev., 28, 121-190, 1981.

Belian, R. D., Gisler, G. R., Cayton, T., and Christensen, R.: High$\mathrm{Z}$ Energetic Particles at Geostationary Orbit During the Great Solar Proton Event Series of October 1989, J. Geophys. Res., 97, 16897-16906, 1992.

Borovsky, J. E., Nemzek, R. J., and Belian, R. D.: The occurrence rate of magnetospheric substorm onsets: random and periodic substorms, J. Geophys. Res., 98, 3807-3813, 1993.

Borovsky, J. E. and Funsten, H. O.: Role of solar wind turbulence in the coupling of the solar wind to the Earth's magnetosphere, J. Geophys. Res., 108(A6), 1246, doi:10.1029/2002JA009601, 2003.

DeJong, A. D., Ridley, A. J., Cai, X., and Clauer, C. R.: A statistical study of BRIs (SMCs), isolated substorms, and individual sawtooth injections, J. Geophys. Res., 114, A08215, doi:10.1029/2008JA013870, 2009.

Frey, H. U., Mende, S. B., Angelopoulos, V., and Donovan, E. F.: Substorm onset observations by IMAGE-FUV, J. Geophys. Res., 109, A10304, doi:10.1029/2004JA010607, 2004.

Gonzalez, W. D., Joselyn, J. A., Kamide, Y., Kroehl, H. W., Rostoker, G., Tsurutani, B. T., and Vasyliunas, V. M.: What is a geomagnetic storm?, J. Geophys. Res., 99, 5771-5792, 1994.

Greenwald, R. A., Baker, K. B., Dudeney, J. R., et al.: DARN/SuperDARN, A global view of the dynamics of highlatitude convection, Space Sci. Rev., 71, 761-796, 1995.

Henderson, M. G., Reeves, G. D., Skoug, R., Thomsen, M. F., Denton, M. H., Mende, S. B., Immel, T. J., Brandt, P. C., and Singer, H. J.: Magnetospheric and auroral activity during the 18 April 2002 sawtooth event, J. Geophys. Res., 111, A01S90, doi:10.1029/2005JA011111, 2006.

Iyemori, T.: Storm-time magnetospheric currents inferred from mid-latitude geomagnetic field variations, J. Geomag. Geoelectr., 42, 1249-1265, 1990.

Kallio, E. I., Pulkkinen, T. I., Koskinen, H. E. J., Viljanen, A., Slavin, J. A., and Ogilvie, K.: Loading-unloading process in the nightside ionosphere, Geophys. Res. Lett., 27, 1627-1630, 2000.

Lui, A. T. Y, Akasofu, S.-I., Hones Jr., E. W., Bame, S. J., and McIlwain, C. E.: Observation of the plasma sheet during a contracted oval substorm in a prolonged quiet period, J. Geophys. Res., 81, 1415-1419, 1976. 
Lyons, L. R., Nagai, T., Blanchard, G. T., Samson, J. C., Yamamoto, T., Mukai, T., Nishida, A., and Kokubun, S.: Association between Geotail plasma flows and auroral poleward boundary intensifications observed by CANOPUS photometers, J. Geophys. Res., 104, 4485-4500, 1999.

Manninen, J., Kleimenova, N. G., Kozyreva, O. V., Ranta, A., Kauristie, K., MŁkinen, S., and Kornilova, T. A.: Ground-based observations during the period between two strong November 2004 storms attributed to steady magnetospheric convection, J. Geophys. Res., 113, A00A09, doi:10.1029/2007JA012984, 2008.

McComas, D. J., Bame, S. J., Parker, P., Feldman, W. C., Phillips, J. L., Riley, P., and Griffee, J. W.: Solar Wind Electron Proton Alpha Monitor (SWEPAM) for the Advanced Composition Explorer, Space Sci. Rev., 86, 563-612, 1998.

McPherron, R. L., O’Brien, T. P., and Thomson, S. M.: Solar wind drivers for steady magnetospheric convection, in Multiscale Coupling of Sun-Earth Processes, 113-124, 2005.

McPherron, R. L., Kepko, L., Pulkkinen, T. I., Hsu, T. S., Weygand, J. W., and Bargatze, L. F.: Changes in the response of the AL Index with solar cycle and epoch within a corotating interaction region, Ann. Geophys., 27, 3165-3178, 2009, http://www.ann-geophys.net/27/3165/2009/.

McWilliams, K. A., Pfeifer, J. B., and McPherron, R. L., Steady magnetospheric convection selection criteria: Implications of global SuperDARN convection measurements, Geophys. Res. Lett., 35, L09102, doi:10.1029/2008GL033671, 2008.

Nevanlinna, H.: Results of the Helsinki magnetic observatory 1844-1912, Ann. Geophys., 22, 1691-1704, 2004, http://www.ann-geophys.net/22/1691/2004/.

O'Brien, T. P., Thompson, S. M., and McPherron R. L.: Steady magnetospheric convection: Statistical signatures in the solar wind and AE, Geophys. Res. Lett., 29(7), 1130, doi:10.1029/2001GL014641, 2002.

Partamies, N., Pulkkinen, T. I., Mcpherron, R. L., McWilliams, K., Bryant, C., Tanskanen, E., Singer, H. J., Reeves, G. D, and Thomsen, M. F.: Statistical survey on sawtooth events, SMCs and isolated substorms, Adv. Space Res., 44, 376-384, doi:10.1016/j.asr.2009.03.013, 2009.

Perreault, P. and Akasofu, S.-I.: A study of geomagnetic storms, Geophys. J. R. Astr. Soc., 54, 547-573, 1978.

Petrukovich, A. A., Baumjohann, W., Nakamura, R., Mukai, T., and Troshichev, O. A.: Small substorms: Solar wind input and magnetotail dynamics, J. Geophys. Res., 105, 21109-21118, 2000.

Pulkkinen, T. I., Ganushkina, N. Yu., Tanskanen, E. I., Kubyshkina, M., Reeves, G. D., Russell, C. T., Singer, H. J., and Slavin, J. A.: Magnetospheric current systems during stormtime sawtooth events, J. Geophys. Res., 111, A11S17, doi:10.1029/2006JA011627, 2006.

Pulkkinen, T. I., Partamies, N., McPherron, R. L., Henderson, M., Reeves, G. D., Thomsen, M. F, and Singer, H.: Statistical analysis of stormtime activations and sawtooth events, J. Geophys. Res., 112, A01205, doi:10.1029/2006JA012024, 2007.

Pulkkinen, T. I., Goodrich, C. C. and Lyon, J. G.: Solar wind electric field driving of magnetospheric activity: Is it velocity or magnetic field?, Geophys. Res. Lett., 34, L21101, doi:10.1029/2007GL031011, 2007.

Pytte, T., McPherron, R. L., Hones Jr., E. W., and West Jr., H. I.: Multiple satellite studies of magnetospheric substorms: distinction between polar magnetic substorms and convection-driven negative bays, J. Geophys. Res., 83, 663-679, 1978.

Reeves, G. D.: Energetic particle observations at geosynchronous orbit, in Proceedings of the International Workshop on Magnetic Storms, Rikubetsu, Japan, 1994.

Reeves, G. D., Henderson, M. G., Skoug, R. M., Thomsen, M. F., Borovsky, J. E., Funsten, H. O., C:son Brandt, P., Mitchell, D. J., Jahn, J.-M., Pollock, C. J., McComas, D. J., and Mende, S. B.: IMAGE, POLAR, and geosynchronous observations of substorm and ring current ion injection, in Disturbances in Geospace: The Storm-Substorm Relationship, Geophys. Monogr. Ser., 142, p. 91, AGU, Washington, D.C., 2004.

Rostoker, G., Samson, J. C., Creutzberg, F., Hughes, T. J., McDiarmid, D. R., McNamara, A. G., Vallace Jones, A., Wallis, D. D., and Cogger, L. L.: CANOPUS - A ground based instrument array for remote sensing in the high latitude ionosphere during ISTP/GGS program, Space Sci. Rev., 71, 743-760, 1995.

Ruohoniemi, J. M. and Baker, K. B.: Large-scale imaging of highlatitude convection with Super Dual Auroral Radar Network HF radar observations, J. Geophys. Res., 103, 20797-20811, 1998.

Russel, C. T. and McPherron, R. L.: Semiannual variation of geomagnetic activity, J. Geophys. Res., 78, 92-108, 1973.

Sergeev, V. A., Pellinen, R. J., and Pulkkinen, T. I.: Steady magnetospheric convection: a review of recent results, Space Sci. Rev., 75, 551-604, 1996.

Smith, C. W., Heureux, J. L., Ness, N. F., Acuna, M. H., Burlaga, L. F., and Scheifele, J.: The ACE magnetic fields experiment, Space Sci. Rev., 86, 613-632, 1998.

Sugiura, M. and Kamei, T.: Equatorial $D_{s t}$ index 1957-1986, in IAGA bulletin, 40, ISGI Publ. Off., Saint Maur, 1991.

Tanskanen, E., Pulkkinen, T. I., Koskinen, H. E. J., and Slavin, J. A.: Substorm energy budget during low and high solar activity: 1997 and 1999 compared, J. Geophys. Res., 107(A6), 1086, doi:10.1029/2001JA900153, 2002.

Tanskanen, E., Slavin, J. A., Fairfield, D. H., Sibeck, D. G., Gjerloev, J., Mukai, T., Ieda, A., and Nagai, T.: Magnetotail response to prolonged southward IMF $B_{Z}$ intervals: Loading, unloading and continuous magnetospheric dissipation, J. Geophys. Res., 110, A03216, doi:10.1029/2004JA010561, 2005.

Thomsen, M. F.: Why $\mathrm{Kp}$ is such a good measure of the magnetospheric convection, Space Weather, 2, S11004, doi:10.1029/2004SW000089, 2004.

Thomsen, M. F., McComas, D. J., Reeves, G. D., and Weiss, L. A.: An observational test of the Tsyganenko (T89a) model of the magnetospheric field, J. Geophys. Res., 101, 24827-24836, 1996.

Troshichev, O. A., Kuznetsov, B. M., and Dmitrieva, N. P.: Polar cap magnetic activity as a signature of substorm development, Planet. Space Sci., 27, 217-221, 1979.

Troshichev, O. A., Lukianova, R. Yu.,Papitashvili, V. O., Rich, F. J., and Rasmussen, O.: Polar cap index (PC) as a proxy for ionospheric electric field in the near-pole region, Geophys. Res. Lett., 27, 3809-3812, 2000.

Turner, N. E., Cramer, W. D., Earles, S. K., and Emery, B. A.: Geoefficiency and energy partitioning in CIR-driven and CMEdriven storms, J. Atmos. Terr. Phys., 71, 1023-1031, 2009.

Viljanen, A. and Häkkinen, L.: IMAGE magnetometer network. In Satellite-Ground Based Coordination Sourcebook, ESA publications SP-1198, 111-117, 1997.

Weimer, D.: Models of high-latitude electric potentials derived with 
a least error fit of spherical harmonic coefficient, J. Geophys. Res., 100, 19595-19608, 1995.

Yahnin, A. G., Malkov, M. V., Sergeev, V. A., Pellinen, R. J., Aulamo, O., Vennerström, S., Friis-Christensen, E., Lassen, K., Danielsen, C., Craven, J. D., Deehr, C., and Frank, L. A.: Features of steady magnetospheric convection, J. Geophys. Res., 99, 4039-4051, 1994.
Zesta, E., Loyns, L. R., and Donovan, E.: The auroral signature of earthward flow bursts observed in the magnetotail, Geophys. Res. Lett., 27, 3241-3244, 2000. 